\title{
Extension and simple Proof of Lekner's Summation Formula for Coulomb Forces
}

\section{Report}

Author(s):

Sperb, René

Publication date:

1993-10

Permanent link:

https://doi.org/10.3929/ethz-a-004284027

Rights / license:

In Copyright - Non-Commercial Use Permitted

Originally published in:

SAM Research Report 1993-06 


\title{
Extension and simple Proof of Lekner's Summation Formula for Coulomb Forces
}

\author{
R. Sperb
}

Research Report No. 93-06

October 1993

Seminar für Angewandte Mathematik

und Institut für Polymere

Eidgenössische Technische Hochschule

CH-8092 Zürich

Switzerland 


\title{
Extension and simple Proof of Lekner's Summation Formula for Coulomb Forces
}

\author{
R. Sperb \\ Seminar für Angewandte Mathematik \\ und Institut für Polymere \\ Eidgenössische Technische Hochschule \\ CH-8092 Zürich \\ Switzerland
}

Research Report No. 93-06 October 1993

\begin{abstract}
A summation formula is proven for a lattice sum occurring frequently in molecular dynamics calculations. It has a much faster convergence rate than the original sum. An important application is in the case of Coulomb forces.
\end{abstract}

Keywords: Coulomb forces, lattice sums, molecular dynamics Subject Classification: $82 \mathrm{~B} 80,82 \mathrm{C} 22$ 
forces. In his derivation he used some powerful identities like the Poisson-Jacobi identity for example, the application of which was not straightforward at all. In this note it is shown that Lekner's result and an extension to more general potentials can be obtained in a rather simple and straightforward way.

\section{A summation formula for forces derived from a power-law potential}

We consider the same situation as Lekner: $N$ particles in a central cell interacting with forces derived from a potential containing only powers of the distance: the force on particle \# $i$ due to particle ${ }^{\#} j$ and all periodic repetitions of $\# j$ is assumed to be of the form

$$
\mathbf{F}_{i}=q_{i} q_{j} \cdot p \sum_{\text {all cells }} \frac{\mathbf{r}_{i}-\mathbf{r}_{j}}{\left|\mathbf{r}_{i}-\mathbf{r}_{j}\right|^{p+2}}
$$

where $\mathbf{r}_{i}-\mathbf{r}_{j}$ are position vectors, $q_{i}, q_{j}$ may be charges, and $p \geq 1$. The "cells" are periodic repetitions of a central cell. We proceed as in [3] and compute only one component of the force since the other components have completely analogous expressions. We set

$$
x_{i}-x_{j}=\xi \cdot L, \quad y_{i}-y_{j}=\eta \cdot L, \quad z_{i}-z_{j}=\zeta \cdot L,
$$

where $L$ is the length of the side of the central cell parallel to the $x$-direction. Note that the cell may be any rectangular parallelepiped (not necessarily a cube). Then the $x$-component of the force is $p q_{i} q_{j} / L^{p+1}$ times $X(\xi, \eta, \zeta)$ where

$$
X(\xi, \eta, \zeta)=\sum_{\ell, m, n=-\infty}^{\infty} \frac{\xi+\ell}{\left[(\xi+\ell)^{2}+\alpha^{2}(\eta+m)^{2}+\beta^{2}(\zeta+n)^{2}\right]^{\frac{p}{2}+1}} .
$$

Here $0 \leq \xi \leq 1$ and $0 \leq \eta \leq \eta_{1}, 0 \leq \zeta \leq \zeta_{1}$, and $\eta_{1}, \zeta_{1}$ are arbitrary positive numbers, and $\alpha=\frac{L_{y}}{L}, \beta=\frac{L_{z}}{L}$, where $L_{y}, L_{z}$ are the corresponding lengths. In order to derive a summation formula with fast convergence for $X(\xi, \eta, \zeta)$ we consider the function

$$
g_{p}(r, \xi):=\sum_{\ell=-\infty}^{\infty} \frac{\xi+\ell}{\left[(\xi+\ell)^{2}+r^{2}\right]^{\frac{p}{2}+1}} .
$$

The function $g_{p}(r, \xi)$ has the properties

(a) $g_{p}(r, \xi+n)=g_{p}(r, \xi), \quad n \in \mathbb{Z}$,

(b) $g_{p}(r,-\xi)=-g_{p}(r, \xi)$,

which follows immediately by replacing the summation index $\ell$ in (4) by $\ell+n$ or $-\ell$ respectively. As a consequence it follows that 
and from (a), (b) combined:

(d) $g_{p}\left(r, \frac{1}{2}\right)=-g_{p}\left(r, \frac{1}{2}-1\right)=0$.

Since $g_{p}(r, \xi)$ is defined for any $r>0$ and vanishes for $\xi=0$ and $\xi=\frac{1}{2}$ we can expand $g_{p}(r, \xi)$ in a Fourier series:

$$
g_{p}(r, \xi)=2 \sum_{k=1}^{\infty} C_{k}^{p}(r) \sin (2 \pi k \xi)
$$

and

$$
C_{k}^{p}(r)=2 \int_{0}^{\frac{1}{2}} \sum_{\ell=-\infty}^{\infty} \frac{\xi+\ell}{\left[(\xi+\ell)^{2}+r^{2}\right]^{\frac{p}{2}+1}} \sin (2 \pi k \xi) d \xi
$$

We may interchange integration and summation. An integration by parts then gives

$$
C_{k}^{p}(r)=\frac{2}{p} \sum_{\ell=-\infty}^{\infty} 2 \pi k \int_{0}^{\frac{1}{2}} \frac{\cos (2 \pi k \xi)}{\left[(\xi+\ell)^{2}+r^{2}\right]^{\frac{p}{2}}} d \xi
$$

We choose a new integration variable $s=\xi+\ell$. This leads to

$$
C_{k}^{p}(r)=\frac{4 \pi k}{p} \sum_{\ell=-\infty}^{\infty} \int_{\ell}^{\ell+\frac{1}{2}} \frac{\cos (2 \pi k s)}{\left[s^{2}+r^{2}\right]^{\frac{p}{2}}} d s .
$$

Since $\cos (2 \pi k s)$ is symmetric in $s$ with period 1 we arrive at

$$
\begin{aligned}
C_{k}^{p}(r) & =\frac{4 \pi k}{p} \int_{0}^{\infty} \frac{\cos (2 \pi k s)}{\left[s^{2}+r^{2}\right]^{\frac{p}{2}}} d s \\
& =\frac{4 \pi k}{p} \cdot\left(\frac{\pi k}{r}\right)^{\frac{p-1}{2}} \frac{\pi^{\frac{1}{2}}}{\Gamma\left(\frac{p}{2}\right)} K_{\frac{p-1}{2}}(2 \pi k r), \quad K=\text { modified Bessel function },
\end{aligned}
$$

where the last integral can be looked up in any table on Fourier-transforms (see e.g. [1], p. 376). Inserting the last expression into (5) leads to

$$
g_{p}(r, \xi)=\frac{8 \pi^{\frac{p}{2}+1}}{p \cdot \Gamma\left(\frac{p}{2}\right)} \sum_{k=1}^{\infty} k\left(\frac{k}{r}\right)^{\frac{p-1}{2}} \cdot K_{\frac{p-1}{2}}(2 \pi k r) \sin (2 \pi k \xi) .
$$

Hence the extension of Lekner's formula (17) to arbitrary powers $p \geq 1$ reads

$$
X(\xi, \eta, \zeta)=\frac{8 \pi^{\frac{p}{2}+1}}{p \cdot \Gamma\left(\frac{p}{2}\right)} \sum_{\ell=1}^{\infty} \ell \cdot \sin (2 \pi \ell \xi) \cdot \sum_{n, m=-\infty}^{\infty}\left(\frac{\ell}{r_{m n}}\right)^{\frac{p-1}{2}} K_{\frac{p-1}{2}}\left(2 \pi \ell \cdot r_{m n}\right),
$$

where $r_{m n}=\left[\alpha^{2}(\eta+m)^{2}+\beta^{2}(\xi+n)^{2}\right]^{\frac{1}{2}}$. 


$$
X(\xi, \eta, \zeta)=8 \pi \sum_{\ell=1}^{\infty} \ell \cdot \sin (2 \pi \ell \xi) \cdot \sum_{n, m=-\infty}^{\infty} K_{0}\left(2 \pi \ell r_{m n}\right) .
$$

For $K_{0}(s)$ approximation formulas can be found e.g. in [1], p. 379.

\section{Discussion}

(a) An important point is to realize that in fact the derivation of the summation formula given here can be extended to general potentials. Suppose that instead of a power law we have a potential $P\left(\left|\mathbf{r}_{i}-\mathbf{r}_{j}\right|\right)$, where the function $P(s)$ satisfies $\lim _{s \rightarrow \infty} P(s)=0$ and $\frac{d P}{d s}=-F(s)$, where $F(s)$ is such that the infinite sum corresponding to $(4)$, namely

$$
g(r, \xi):=\sum_{\ell=-\infty}^{\infty} \frac{(\xi+\ell)}{\left((\xi+\ell)^{2}+r^{2}\right)^{1 / 2}} F\left(\left[(\xi+\ell)^{2}+r^{2}\right]^{1 / 2}\right)
$$

still converges.

One can check that all steps leading to the formulas (10) and (11) can be repeated similarly. One just has to replace the Fourier-coefficient in (9) by

$$
C_{k}(r)=4 \pi k \int_{0}^{\infty} P\left(\left[s^{2}+r^{2}\right]^{1 / 2}\right) \cdot \cos (2 \pi k s) d s
$$

In a number of examples the expression $C_{k}(r)$ can be given explicitly. As an example let us take

$$
P(s)=e^{-\beta s} \text { for some } \beta>0
$$

Then one has (see [2])

$$
C_{k}(r)=4 \pi k r \beta\left(4 \pi^{2} k^{2}+\beta^{2}\right)^{-1 / 2} K_{1}\left[r\left(4 \pi^{2} k^{2}+\beta^{2}\right)^{1 / 2}\right]
$$

and formula (11) has to be changed in an obvious way.

(b) The convergence in formula (10) is slow if $r$ is less than 0.1. It suffices therefore to give an alternative to (11) only for $n=m=0$. We go back to (4) which can be written as

$$
g_{p}(r, \xi)=\frac{\xi}{\left(r^{2}+\xi^{2}\right)^{\frac{p+2}{2}}}+\sum_{\ell=1}^{\infty} \frac{\ell+\xi}{\left(r^{2}+(\ell+\xi)^{2}\right)^{\frac{p+2}{2}}}-\sum_{\ell=1}^{\infty} \frac{\ell-\xi}{\left(r^{2}+(\ell-\xi)^{2}\right)^{\frac{p+2}{2}}}
$$




$$
\begin{aligned}
\frac{\ell+\xi}{\left(r^{2}+(\ell+\xi)^{2}\right)^{\frac{p+2}{2}}} & =\frac{1}{(\ell+\xi)^{p+1}} \frac{1}{\left(1+\left(\frac{r}{\ell+\xi}\right)^{2}\right)^{\frac{p+2}{2}}} \\
& =\frac{1}{(\ell+\xi)^{p+1}} \cdot \sum_{k=0}^{\infty}\left(\begin{array}{c}
-\frac{p+2}{2} \\
k
\end{array}\right) r^{2 k} \frac{1}{(\ell+\xi)^{2 k}} \\
& =\sum_{k=0}^{\infty}\left(\begin{array}{c}
-\frac{p+2}{2} \\
k
\end{array}\right) r^{2 k} \frac{1}{(\ell+\xi)^{2 k+p+1}}
\end{aligned}
$$

We now use the Hurwitz Zeta function $\zeta(m, \xi)$ (see e.g. [1] Eq. 6.4.10, p.220), which is just a multiple of the Polygamma function $\psi^{m-1}$ in our case:

$$
\zeta(m, \xi)=\sum_{\ell=0}^{\infty} \frac{1}{(\ell+\xi)^{m}}=(-1)^{m} \frac{1}{(m-1) !} \cdot \psi^{m-1}(\xi)
$$

It is easy to check that the expression for $g_{p}(r, \xi)$ becomes

$$
g_{p}(r, \xi)=\frac{\xi}{\left(r^{2}+\xi^{2}\right)^{\frac{p+2}{2}}}+\sum_{k=0}^{\infty}\left(\begin{array}{c}
-\frac{p+2}{2} \\
k
\end{array}\right) r^{2 k}\{\zeta(2 k+1+p, \xi)-\zeta(2 k+p+1,-\xi)\}
$$

Numerical tests show that the sum in formula (18) converges very fast if $r<0.2$.

(c) The function $g_{1}(r, \xi)$ is a radially symmetric (with respect to $r$ ) solution of

$\Delta u=0$ for $r>0, \xi \in \mathbb{R}$, $u(r, 0)=u\left(r, \frac{1}{2}\right)=0$, $\lim _{r \rightarrow \infty} u(r, \xi)=0$.

Separation of variables then shows that any solution $u(r, \xi)$ can be written in the form

$$
u(r, \xi)=\sum_{\ell=1}^{\infty} C_{\ell} K_{0}(2 \pi \ell r) \sin (2 \pi \ell \xi),
$$

for any $C_{\ell}$ for which series converges.

For $C_{\ell}=8 \pi \cdot \ell$ one is led to Lekner's summation formula.

(d) For $p=2,4,6, \ldots$ the Bessel functions appearing in (11) can be written in terms of elementary expressions. One has (see e.g. [1])

$$
K_{n+\frac{1}{2}}(s)=\sqrt{\frac{\pi}{2 s}} e^{-s} f_{n}(s),
$$




$$
f_{n+1}(s)=\frac{2 n+1}{s} f_{n}(s)+f_{n-1}(s) .
$$

For example for $p=6$ and $p=12$ as is the case for the Lennard-Jones potential one would need in (11)

$$
K_{\frac{5}{2}}(s)=\sqrt{\frac{\pi}{2 s}} e^{-s}\left(1+\frac{3}{s}+\frac{3}{s^{2}}\right)
$$

and

$$
K_{\frac{11}{2}}(s)=\sqrt{\frac{\pi}{2 s}} e^{-s} \cdot\left(1+\frac{15}{s}+\frac{105}{s^{2}}+\frac{1120}{s^{3}}+\frac{945}{s^{4}}+\frac{945}{s^{5}}\right) .
$$

(e) As a simple approximation for $g_{p}(r, \xi)$ one could replace the sum in (10) by an integral

$$
g_{p}(r, \xi) \cong \frac{8 \pi^{\frac{p}{2}+1}}{p \cdot \Gamma\left(\frac{p}{2}\right)} \int_{0}^{\infty} k\left(\frac{k}{r}\right)^{\frac{p-1}{2}} \cdot K_{\frac{p-1}{2}}(2 \pi k r) \sin (2 \pi k \xi) d k .
$$

After some simplification one gets (see [2])

$$
g_{p}(r, \xi) \cong \frac{\xi}{\left(\xi^{2}+r^{2}\right)^{\frac{p}{2}+1}},
$$

that is, the approximation of the sum in (10) by an integral leads back to the term for $\ell=0$ in (4)!

(f) In order to illustrate the speed-up of convergence given by formulas (10), (11) consider unit charges at points $0, \pm 1, \pm 2, \ldots$ on the $\xi$-axis. Then in order to calculate the Coulomb force, say at $\xi=0.2$ and distance 1 from the $\xi$-axis, directly, one needs 3600 terms to get seven digits accuracy. Using formula (10) in this case we get the same accuracy with only two terms!

(g) It will be shown in a forthcoming paper how the energy can be calculated as well by our method ( $N$ charges in the central cell, assuming charge neutrality). 
[1] M. Abramowitz \& I. Stegun: Handbook of Mathematical functions, Dover, New York (1970).

[2] Tables of Integral Transforms, Vol, I, Bateman Manuscript Project, McGraw Hill (1954).

[3] J. Lekner: Summation of Coulomb Fields in Computer-Simulated Disordered Systems, Physica A 176 (1991), 485-498. 
93-06

R. Sperb

93-05

A. Frommer, B. Pohl

93-04 M. Pirovino

93-03 R. Jeltsch, X. Wang

93-02 W.-A. Yong

93-01 Ch. Lubich, K. Nipp, D. Stoffer

92-15 N. Botta

92-14 K. Nipp, D. Stoffer

92-13 K. Nipp

92-12 D. Мao

92-11 K. Nipp, D. Stoffer

92-10 M. Fey, R. Jeltsch

92-09 M. Fey, R. Jeltsch

92-08 M. Fey, R. Jeltsch, P. Karmann

92-07 M. Fey, R. Jeltsch, P. Karmann

92-06 M. Fey, R. Jeltsch, S. Müller

92-05 N. Botta, J. Sesterhenn

92-04 Ch. Lubich

92-03 M. Fey, R. Jeltsch, S. Müller
Extension and simple Proof of Lekner's Summation Formula for Coulomb Forces

A Comparison Result for Multisplittings Based on Overlapping Blocks and its Application to Waveform Relaxation Methods

The Inverse Sturm-Liouville Problem and Finite Differences

Uniqueness of Piecewise Lipschitz Continuous Solutions of the Cauchy-Problem for $2 \times 2$ Conservation Laws

Difference approximations to the global $W^{1, \infty}$ solutions of the isentropic gas equations

Runge-Kutta solutions of stiff differential equations near stationary points

Is the transonic flow around a cylinder always periodic?

Invariant manifolds of numerical integration schemes applied to stiff systems of singular perturbation type - Part I: $R K$-methods Smooth attractive invariant manifolds of singularly perturbed ODE's

A Shock Tracking Technique Based on Conservation in One Space Dimension

Attractive invariant manifolds for maps:

Existence, smoothness and continuous depen-

dence on the map

A Simple Multidimensional Euler Scheme

A New Multidimensional Euler Scheme

Numerical solution of a nozzle flow

Special aspects of reacting inviscid blunt body flow

The influence of a source term, an example: chemically reacting hypersonic flow

Deficiencies in the numerical computation of nozzle flow

Integration of stiff mechanical systems by Runge-Kutta methods

Stagnation point analysis 